\title{
THE GENUS OF REPEATED CARTESIAN PRODUCTS OF BIPARTITE GRAPHS $\left(^{(}\right)$
}

\author{
BY \\ ARTHUR T. WHITE
}

\begin{abstract}
With the aid of techniques developed by Edmonds, Ringel, and Youngs, it is shown that the genus of the cartesian product of the complete bipartite graph $K_{2 m, 2 m}$ with itself is $1+8 m^{2}(m-1)$. Furthermore, let $Q_{1}^{(s)}$ be the graph $K_{s, s}$ and recursively define the cartesian product $Q_{n}^{(s)}=Q_{n-1}^{(s)} \times K_{s, s}$ for $n \geqq 2$. The genus of $Q_{n}^{(s)}$ is shown to be $1+2^{n-3} s^{n}(s n-4)$, for all $n$, and $s$ even; or for $n>1$, and $s=1$ or 3 . The graph $Q_{n}^{(1)}$ is the 1-skeleton of the $n$-cube, and the formula for this case gives a result familiar in the literature. Analogous results are developed for repeated cartesian products of paths and of even cycles.
\end{abstract}

Introduction. In this paper a graph $G$ is a finite 1-complex. The genus $\gamma(G)$ of $G$ is the minimum genus among the genera of all compact orientable 2-manifolds in which $G$ can be imbedded. All 2-manifolds in this paper are assumed to be compact and orientable. There are very few families of graphs for which the genus has been determined; these include the complete graphs (Ringel and Youngs [7]), the complete bipartite graphs, (Ringel [5]), and some subfamilies of the family of complete tripartite graphs (see [6] and [8]).

One of the first genus formulae was developed by Ringel [4] in 1955 (and independently by Beineke and Harary [1] in 1965) when he found that the genus of the $n$-cube $Q_{n}$ is given by:

$$
\gamma\left(Q_{n}\right)=1+2^{n-3}(n-4), \text { for } n \geqq 2 .
$$

The $n$-cube can be defined as a repeated cartesian product: let $Q_{1}=K_{2}$, the complete graph on two vertices, and recursively define $Q_{n}=Q_{n-1} \times K_{2}$ for $n \geqq 2$. In general, given two graphs $G_{1}$ and $G_{2}$, with vertex sets $V\left(G_{1}\right), V\left(G_{2}\right)$ and edge sets $E\left(G_{1}\right)$, $E\left(G_{2}\right)$ respectively, the cartesian product $G_{1} \times G_{2}$ is formed by taking $V\left(G_{1} \times G_{2}\right)$ $=\left\{\left(u_{1}, u_{2}\right): u_{1} \in V\left(G_{1}\right), u_{2} \in V\left(G_{2}\right)\right\}$ and $E\left(G_{1} \times G_{2}\right)=\left\{\left[\left(u_{1}, u_{2}\right),\left(v_{1}, v_{2}\right)\right]: u_{1}=v_{1}\right.$

Received by the editors October 6, 1969.

AMS Subject Classifications. Primary 0550; Secondary 5475, 5510.

Key Words and Phrases. Genus of a graph, cartesian product of two graphs, bipartite graph, complete bipartite graph, path, cycle, $n$-cube, 2-manifold, Euler formula, Edmonds' permutation technique, quadrilateral imbedding, cyclomatic number of a graph.

(1) Definitions not presented in this article may be found in [3]. This research was supported in part by National Science Foundation Grant GZ 1222. The results formed part of the author's Ph.D. thesis, written at Michigan State University. The author wishes to express his gratitude to Professor E. A. Nordhaus for his guidance during this research.

Copyright (C) 1970, American Mathematical Society 
and $\left[u_{2}, v_{2}\right] \in E\left(G_{2}\right)$ or $u_{2}=v_{2}$ and $\left.\left[u_{1}, v_{1}\right] \in E\left(G_{1}\right)\right\}$. In this paper we develop genus formulae for three families of graphs, all of which are defined in terms of repeated cartesian products of bipartite graphs. Two of these families include all of the $n$-cubes, while the third family includes the $2 n$-cubes.

Some elementary results on the genus of cartesian products of bipartite graphs. The following two propositions are employed in computing each of the genus results developed in this article; the proofs are straightforward and will be omitted.

Proposition 1. The cartesian product of two bipartite graphs is bipartite.

It follows by a routine application of mathematical induction that if the graphs $G_{i}$ are bipartite, $i=1,2, \ldots$, so are the graphs $H_{n}$, where $H_{1}=G_{1}$ and $H_{n}=H_{n-1}$ $\times G_{n}$, for $n \geqq 2$. A quadrilateral imbedding of a graph $G$ is an imbedding for which every face has four sides.

Proposition 2. If the bipartite graph $G$ with $V$ vertices and $E$ edges has a quadrilateral imbedding, then that imbedding is minimal, and $\gamma(G)=1+E / 4-V / 2$.

It will therefore suffice to produce a quadrilateral imbedding for each of the graphs under consideration, as the genus may then be computed directly using Proposition 2.

The constructions employed in this article to produce quadrilateral imbeddings of $G_{1} \times G_{2}$ will begin with $V_{2}$ copies of $G_{1}$ (where $G_{2}$ has $V_{2}$ vertices) minimally imbedded in $V_{2}$ 2-manifolds, and the necessary additional edges will be added over tubes added between the 2-manifolds.

The addition of a tube to a generalized 2-manifold. By a generalized 2-manifold, $M$, is meant a finite collection of compact orientable 2-manifolds in Euclidean 3-space, each of which is exterior to all the others. Let $C_{1}$ and $C_{2}$ be two disjoint simple closed curves on $M$ such that $C_{1}$ is homotopic zero on $M-C_{2}$, and $C_{2}$ is homotopic to zero on $M-C_{1}$. Remove two open disks from $M$ having $C_{1}$ and $C_{2}$ as their respective boundaries. Then the process of adding a tube to $M$ is to adjoin a topological cylinder $K$ with bases $C_{1}$ and $C_{2}$ such that $K \cap M=C_{1} \cup C_{2}$. That this may indeed be done can be established by standard topological arguments; the proof is omitted. In the context of this paper, $C_{1}$ and $C_{2}$ will always be in the respective interiors of corresponding faces of mirror image imbeddings of the same graph in two 2-manifolds of identical genus. The tube will then be used to carry one edge for each pair of corresponding vertices in the two faces. That this may be accomplished without the edges intersecting one another follows from the fact that the faces are mirror images of each other.

Repeated cartesian products of complete bipartite graphs. In this section we compute the genus of the graph $K_{s, s} \times K_{s, s}$ for the cases $s=1, s=3$, and for all even $s$. We then generalize this result by taking the cartesian product of arbitrarily many copies of $K_{s, s}$ and computing the genus of the resulting graph. 
For $s=2 m$, Ringel [5] has shown that $\gamma\left(K_{2 m, 2 m}\right)=(m-1)^{2}$, with $F=F_{4}=2 m^{2}$; that is, every face is a quadrilateral. The imbedding given by Ringel for this graph may be presented in the following manner. Designate the vertex set of $K_{2 m, 2 m}$ by $V\left(K_{2 m, 2 m}\right)=\{1, \ldots, 4 m\}$, with adjacencies at vertex $i$ given by

$$
\begin{aligned}
V(i) & =\{j: 2 m+1 \leqq j \leqq 4 m\}, & & 1 \leqq i \leqq 2 m, \\
& =\{j: 1 \leqq j \leqq 2 m\}, & & 2 m+1 \leqq i \leqq 4 m .
\end{aligned}
$$

Define cyclic permutations $P_{i}: V(i) \rightarrow V(i)$, for $i=1, \ldots, 4 m$ by:

$$
\begin{aligned}
& P_{1}, P_{3}, \ldots, P_{2 m-1}:(2 m+1,2 m+2, \ldots, 4 m), \\
& P_{2}, P_{4}, \ldots, P_{2 m}:(4 m, 4 m-1, \ldots, 2 m+1), \\
& P_{2 m+1}, P_{2 m+3}, \ldots, P_{4 m-1}:(1,2, \ldots, 2 m), \\
& P_{2 m+2}, P_{2 m+4}, \ldots, P_{4 m}:(2 m, 2 m-1, \ldots, 1) .
\end{aligned}
$$

It is a theorem of Edmonds ([2], see also Youngs [9]) that the collection $\left(P_{1}, \ldots\right.$, $\left.P_{4 m}\right)$ uniquely determines a 2-cell imbedding of $K_{2 m, 2 m}$ in a 2-manifold $M$, once an orientation is selected. Furthermore, let $[a, b]$ represent the directed edge from vertex $a$ to vertex $b$ corresponding to edge $(a, b)$ in $K_{2 m, 2 m}$ and form the set $W=\left\{[a, b]:(a, b) \in E\left(K_{2 m, 2 m}\right)\right\}$. Now define the permutation $P: W \rightarrow W$ by $P([a, b])=\left[b, P_{b}(a)\right]$. Then the orbits under $P$ correspond to (2-cell) faces of the imbedding. Had the opposite orientation been selected, with the collection $\left(P_{1}, \ldots, P_{4 m}\right)$ unchanged, the resulting imbedding would have been a mirror image of the first imbedding. The following lemma is used to compute the genus of $K_{2 m, 2 m} \times K_{2 m, 2 m}$ :

LEMMA 1. For the imbedding of $K_{2 m, 2 m}$ given above, the set of $2 m^{2}$ quadrilateral faces may be partitioned into $2 m$ subsets of $m$ faces each so that each subset of $m$ faces contains all $4 m$ vertices of the graph.

Proof. We write out the orbits (each corresponding to a quadrilateral face) determined by the permutation $P$ as defined by the permutations $P_{i}, 1 \leqq i \leqq 4 m$, given above:

$$
\begin{array}{ll}
(2 g-1)-(2 h-1)-2 g-(2 h-2), & 1 \leqq g \leqq m ; m+1<h \leqq 2 m, \\
(2 g-1)-(2 h-1)-2 g-4 m, & 1 \leqq g \leqq m ; h=m+1, \\
2 j-(2 k-1)-(2 j+1)-2 k, & m+1 \leqq k \leqq 2 m, 1 \leqq j<m, \\
2 j-(2 k-1)-1-2 k, & m+1 \leqq k \leqq 2 m, j=m .
\end{array}
$$

We now assign these $2 m^{2}$ faces to parts of the partition. For fixed $i$, the $m$ faces of part $(2 i-1)$ are determined by selecting $h=m+g+i$, with $1 \leqq g \leqq m$, where we reduce $(g+i)$ modulo $m$ and write $m$ instead of 0 . The $m$ faces of part $2 i$ are determined by taking $k=m+j+i$, with $1 \leqq j \leqq m$, where we reduce $(j+i)$ modulo $m$ and again write $m$ instead of 0 . Letting $i$ run between 1 and $m$, we obtain $2 m$ sets of $m$ faces each, the sets being mutually disjoint by the manner in which they were 
selected. Furthermore, each set of $m$ faces contains all $4 m$ vertices of the graph $K_{2 m, 2 m}$.

We are now in a position to prove the following theorem:

THEOREM 1. The genus of $K_{s, s} \times K_{s, s}$ is given by $\gamma\left(K_{s, s} \times K_{s, s}\right)=1+s^{2}(s-2)$, if $s$ is even or if $s=1$ or 3 .

Proof. We consider three cases:

Case (i). For $s=2 m$, imbed $4 m$ copies of $K_{2 m, 2 m}$ in $4 m$ 2-manifolds of genus $(m-1)^{2}$, using the imbedding described above. We choose one of the two possible orientations for $2 m$ of these 2 -manifolds, and the reverse orientation for the remaining $2 m$ 2-manifolds. This partition corresponds to the vertex set partition for $K_{2 m, 2 m}$. Between each pair of oppositely oriented 2-manifolds, we must add $4 m$ edges in order to imbed $K_{2 m, 2 m} \times K_{2 m, 2 m}$. We add these $4 m$ edges over a join composed of $m$ tubes, each tube carrying four edges. Each tube is attached between corresponding faces in imbeddings of opposite orientation, so that the faces are mirror images of each other. There are $2 m$ such joins that must be made from each 2-manifold. Lemma 1 establishes that Ringel's imbedding for the copy of $K_{2 m, 2 m}$ at each 2-manifold is ideally suited for this purpose. We need only check that we can match corresponding parts of the face partitions appropriately. At copy $j$, $1 \leqq j \leqq 2 m$, of $K_{2 m, 2 m}$ minimally imbedded with common orientation, match part $i$ of the face partition with part $i$ in copy $j+i, 1 \leqq j+i \leqq 2 m(\bmod 2 m)$ of $K_{2 m, 2 m}$ minimally imbedded with the opposite orientation. If $\boldsymbol{j}+\boldsymbol{i}=\boldsymbol{j}^{\prime}+\boldsymbol{i}^{\prime}$ with $\boldsymbol{i}=\boldsymbol{i}^{\prime}$, then $j=j^{\prime}$, so that each part of each partition has exactly one tube attached at each face in that part. As each tube carries the maximum of four edges, each new face formed (intersecting some tube) is a quadrilateral. We have thus constructed a quadrilateral imbedding of $K_{2 m, 2 m} \times K_{2 m, 2 m}$, and the genus may be computed using Proposition 2. As $V=16 m^{2}$ and $E=32 m^{3}$, we see that $\gamma\left(K_{2 m, 2 m} \times K_{2 m, 2 m}\right)$ $=1+8 m^{2}(m-1)=1+s^{2}(s-2)$, for $s=2 m$.

Case (ii). For $s=3$, we use an imbedding of $K_{3,3}$ for which $F=F_{6}=3$ :

$$
\begin{aligned}
& V(i)=\{4,5,6\}, \quad i=1,2,3, \\
&=\{1,2,3\}, \quad i=4,5,6, \\
& P_{1}, P_{2}, P_{3}:(4,6,5), \quad P_{4}, P_{5}, P_{6}:(1,3,2) .
\end{aligned}
$$

For this imbedding, each face contains each vertex of the graph exactly once. We imbed six copies of $K_{3,3}$ in six 2-manifolds of genus one (three of each orientation) and add nine tubes (each carrying six edges) in the fashion described above. A quadrilateral imbedding results, and we compute that $\gamma\left(K_{3,3} \times K_{3,3}\right)=10$.

Case (iii). For $s=1, K_{1,1} \times K_{1,1}=K_{2} \times K_{2}=C_{4}$, and $\gamma\left(C_{4}\right)=0$. This completes the proof of the theorem.

For the construction of Case (i) above (as well as for Case (ii)), we can compute the genus of the resulting 2-manifold directly, without recourse to the Euler-type 
formula of Proposition 2. The contributions to the genus are of three types: (i) $4 m \gamma\left(K_{2 m, 2 m}\right)=4 m(m-1)^{2}$, representing the collective genera of the 2-manifolds with which we began our construction; (ii) $4 m^{2}(m-1)$, representing an increase of $(m-1)$ in the genus for each of the $4 m^{2}$ joins, due to the addition of $m$ tubes; and (iii) $\beta\left(K_{2 m, 2 m}\right)=(2 m-1)^{2}$ (where $\beta(G)=E-V+1$ is the cyclomatic number of $G)$, representing the contributions of the joins taken collectively. Adding, we see that

$$
\gamma\left(K_{2 m, 2 m} \times K_{2 m, 2 m}\right)=1+8 m^{2}(m-1) .
$$

We can use Theorem 1 to prove the following corollary, which is actually a generalization of the theorem:

COROLlaRY 1a. The genus of $K_{2 m, 2 m} \times K_{r, s}$ is given by

$$
\gamma\left(K_{2 m, 2 m} \times K_{r, s}\right)=1+m((m-2)(r+s)+r s),
$$

if $r \leqq 2 m$ and $s \leqq 2 m$.

Proof. Imbed $K_{2 m, 2 m} \times K_{2 m, 2 m}$ as in the proof of Theorem 1, with $F=F_{4}$. Remove $(4 m-(r+s))$ 2-manifolds containing copies of $K_{2 m, 2 m}$, together with all tubes and edges issuing from these 2-manifolds, so as to leave an imbedding of $K_{2 m, 2 m} \times K_{r, s}$. This imbedding is also quadrilateral, since each copy of $K_{2 m, 2 m}$ was initially imbedded quadrilaterally, and the removal of any tube reintroduces only quadrilateral faces. Noting that, for $K_{2 m, 2 m} \times K_{r, s}, V=4 m(r+s)$ and $E=4 m^{2}(r+s)+4 m r s$, the result now follows directly from Proposition 2 .

We apply Corollary 1a in turn to give the following unrestricted 2-parameter formula:

Corollary 1b. The genus of $K_{2 m, 2 m} \times K_{2 n, 2 n}$ is given by

$$
\gamma\left(K_{2 m, 2 m} \times K_{2 n, 2 n}\right)=1+4 m n(m+n-2),
$$

for all natural numbers $m$ and $n$.

We now define a class of graphs which generalize the $n$-cube as follows: let $Q_{1}^{(s)}=K_{s, s}$, and recursively define $Q_{n}^{(s)}=Q_{n-1}^{(s)} \times K_{s, s}$, for $n \geqq 2$. The constructions of Theorem 1 can now be extended, as developed below:

THEOREM 2. The genus of $Q_{n}^{(2 m)}$ is given by $\gamma\left(Q_{n}^{(2 m)}\right)=1+2^{2 n-2} m^{n}(m n-2)$.

Proof. By the observation following Proposition $1, Q_{n}^{(2 m)}$ is a bipartite graph. We construct a quadrilateral imbedding for $Q_{n}^{(2 m)}$, and compute $\gamma\left(Q_{n}^{(2 m)}\right)$ using Proposition 2. It is clear that $V=4^{n} m^{n}$ for $Q_{n}^{(2 m)}$. We establish the values of $E$ and $F$, showing that $F=F_{4}$, by mathematical induction. Let the statement $S(n)$ be as follows: There is an imbedding of $Q_{n}^{(2 m)}$ with $E^{(n)}=n 2^{2 n} m^{n+1}$ and $F^{(n)}=F_{4}^{(n)}$ $=n 2^{2 n-1} m^{n+1}$, including $2 m$ mutually disjoint sets of $2^{2 n-2} m^{n}$ mutually vertexdisjoint quadrilateral faces each, each set containing all $4^{n} m^{n}$ vertices of $Q_{n}^{(2 m)}$. 
We claim that $S(n)$ is true for all natural numbers $n$. We establish this claim by induction. That $S(1)$ is true follows immediately from Ringel's imbedding of $K_{2 m, 2 m}$ and Lemma 1 .

Now, assuming $S(n)$ to be true, we establish $S(n+1)$, for $n \geqq 1$. So, consider a large copy of $K_{2 m, 2 m}$, each vertex of which is replaced with a small copy of $Q_{n}^{(2 m)}$ imbedded as described by $S(n)$ and with respective orientations determined by the vertex set partition for $K_{2 m, 2 m}$. Label the $2 m$ copies of one orientation by $j, 1 \leqq j \leqq 2 m$, and the $2 m$ copies of opposite orientation by $j, 1 \leqq j \leqq 2 m$. Now, by the induction hypothesis, each copy of $Q_{n}^{(2 m)}$ has $2 m$ sets of faces available, one set for each of the $2 m$ joins that must be made from that copy. Furthermore, each set contains each vertex of the graph $Q_{n}^{(2 m)}$ exactly once. As in the proof of Theorem 1, at copy $j$, $1 \leqq j \leqq 2 m$, match set $i$ with set $i$ in copy $j+i, 1 \leqq j+i \leqq 2 m(\bmod 2 m)$. For each matching a tube carrying four edges is attached between each pair of corresponding quadrilateral faces. In this manner the required $4 \mathrm{~m}^{2}$ joins are completed, so that we have a quadrilateral imbedding of $Q_{n+1}^{(2 m)}$.

Now, for fixed $j$, pair off copy $i$ of $Q_{n}^{(2 m)}$ with copy $i+j$, where $\mathbf{1} \leqq i+j \leqq 2 m$ $(\bmod 2 m)$. For each such pairing, with copy $i$ joined to copy $i+j$ by $2^{2 n-2} m^{n}$ tubes, we have (for fixed $j$ and $i=1, \ldots, 2 m)$ a total of $4\left(2^{2 n-2}\right) m^{n}(2 m)=2^{2 n+1} m^{n+1}$ quadrilateral faces on $2^{2 n-1} m^{n+1}$ tubes. For each tube, select one pair of opposite faces. The $2^{2 n} m^{n+1}$ faces thus selected are mutually vertex-disjoint and contain all $4^{n+1} m^{n+1}$ vertices of $Q_{n+1}^{(2 m)}$. Now letting $j$ vary between 1 and $2 m$, we obtain $2 m$ mutually disjoint such sets of quadrilateral faces, as claimed by $S(n+1)$.

The imbedding of $Q_{n+1}^{(2 m)}$ we have obtained has $F^{(n+1)}=F_{4}^{(n+1)}$, since $F^{(n)}=F_{4}^{(n)}$ and the attaching of each new tube with the four edges it carries eliminates two quadrilaterals and introduces four new quadrilaterals. Now, $F^{(n+1)}=4 m F^{(n)}+\Delta F$, where $\Delta F$ is twice the number of tubes added at this stage. But the number of tubes added is $\left(4 m^{2}\right)\left(4^{n} m^{n}\right) / 4=4^{n} m^{n+2}$, where $4 m^{2}$ is the number of edges in $K_{2 m, 2 m}$ (corresponding to the number of joins we made), $4^{n} m^{n}$ is the number of edges per join, and there are four edges per tube. Hence

$$
\begin{aligned}
F^{(n+1)} & =4 m\left(n 2^{2 n-1} m^{n+1}\right)+2\left(4^{n} m^{n+2}\right) \\
& =(n+1) 2^{2 n+1} m^{n+2} .
\end{aligned}
$$

Also,

$$
\begin{aligned}
E^{(n+1)} & =4 m E^{(n)}+4 m^{2} V^{(n)} \\
& =4 m\left(n 2^{2 n} m^{n+1}\right)+4 m^{2}\left(4^{n} m^{n}\right) \\
& =(n+1) 2^{2 n+2} m^{n+2} .
\end{aligned}
$$

We have established that $S(n+1)$ follows from $S(n)$, for all $n \geqq 1$. Thus $S(n)$ holds, for all natural numbers $n$. Now by Proposition 2,

$$
\begin{aligned}
\gamma\left(Q_{n}^{(2 m)}\right) & =1+n 2^{2 n} m^{n+1} / 4-4^{n} m^{n} / 2 \\
& =1+2^{2 n-2} m^{n}(m n-2) .
\end{aligned}
$$


In Theorem 2, it was convenient to consider $K_{s, s}$ for $s$ even, since $K_{2 m, 2 m}$ has $F=F_{4}$ in its minimal imbedding. The arguments of Theorem 2, with minor modifications, apply also to the cases $s=1$ and $s=3$. We can therefore state:

TheOREM 3. The genus of $Q_{n}^{(s)}$ is given by $\gamma\left(Q_{n}^{(s)}\right)=1+2^{n-3} s^{n}(n s-4)$, for $s$ even and any positive integer $n$, or for $s=1$ or 3 and $n \geqq 2$.

The genus formula given in Theorem 3 includes as two of its special cases $\gamma\left(K_{2 m, 2 m}\right)=(m-1)^{2}$ (for $n=1, s=2 m$ ) and $\gamma\left(Q_{n}\right) 1+2^{n-3}(n-4)$ (for $s=1$, since $K_{1,1}=K_{2}$ ), two of the familiar results in the literature.

It was crucial to the construction of imbeddings for $G_{1} \times G_{2}$ employed in this section that both $G_{1}$ and $G_{2}$ be bipartite, for then Proposition 2 may be employed, due to Proposition 1. Moreover, in commencing our construction with $V_{2} 2$ manifolds partitioned by orientation in accordance with the vertex-set partition of $G_{2}$, we are assured that every tube to be added in the construction will be attached at two faces of opposite orientation, so that the required edges can be added without intersection. In the next two sections, we take advantage of this situation for two different families of bipartite graphs.

Repeated cartesian products of paths. We define a second class of graphs generalizing the $n$-cube by: $H_{1}=P_{m_{1}}$, a path on $m_{1}$ vertices, and $H_{n}=H_{n-1} \times P_{m_{n}}$, for $n \geqq 2$. All paths are bipartite graphs, but we nevertheless restrict $m_{1}, m_{2}$, and $m_{3}$ to be even in the theorem to follow. Let

$$
M^{(n)}=\prod_{i=1}^{n} m_{i} \quad \text { and } \quad m^{(n)}=\sum_{i=1}^{n} \frac{1}{m_{i}} .
$$

THEOREM 4. The genus of $H_{n}$ is given by $\gamma\left(H_{n}\right)=1+\left(M^{(n)} / 4\right)\left(n-2-m^{(n)}\right)$, for $n \geqq 3$ and $m_{1}, m_{2}$, and $m_{3}$ all even.

Proof. By the observation following Proposition $1, H_{n}$ is bipartite. We construct a quadrilateral imbedding for $H_{n}$, and compute $\gamma\left(H_{n}\right)$ using Proposition 2. For $H_{n}, V^{(n)}=M^{(n)}$. Let the statement $S^{(n)}$ be: There is an imbedding of $H_{n}$ for which $F^{(n)}=F_{4}^{(n)}=\left(M^{(n)} / 2\right)\left(n-m^{(n)}\right)$, including two disjoint sets of $\frac{1}{4} M^{(n)}$ mutually vertexdisjoint quadrilateral faces each, both sets containing all $M^{(n)}$ vertices of $H_{n}$; furthermore, for $H_{n}, E^{(n)}=M^{(n)}\left(n-m^{(n)}\right)$. We claim that $S(n)$ is true for all $n \geqq 3$. We verify this by induction.

To see that $S(3)$ is true, refer to Figure 1 , which shows that $\gamma\left(P_{6} \times P_{8}\right)=0$. Every face but the exterior is a quadrilateral for this imbedding. We see that two joins may be made at each copy of $P_{m_{1}} \times P_{m_{2}}$ in general, provided $m_{1}$ and $m_{2}$ are both even. One join employs the faces designated by (1), and the other join uses the faces designated by (2), as in Figure 1. Provided $m_{3}$ is even also, we can arrange the two end copies of $P_{m_{1}} \times P_{m_{2}}$ so that the faces (2), including the exterior face, are employed in the single join that must be made from each end copy. Partition the $m_{3}$ copies of $P_{m_{1}} \times P_{m_{2}}$ into $m_{3} / 2$ copies of one orientation and $m_{3} / 2$ copies of 


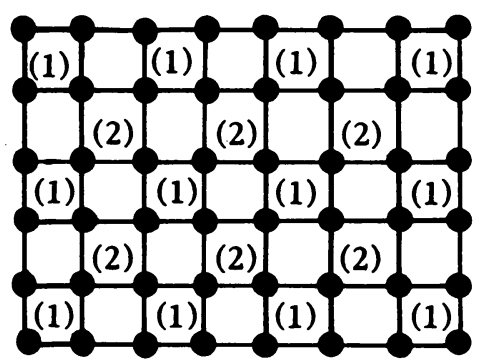

Figure 1. An imbedding of $P_{6} \times P_{8}$ in the plane

the other orientation, with the two end copies in different parts of this partition (corresponding to the vertex set partition of $P_{m_{3}}$ ). The graph $H_{3}=P_{m_{1}} \times P_{m_{2}} \times P_{m_{3}}$ thus has a quadrilateral imbedding, since a tube attached between two oppositely oriented copies of the exterior face (2) replaces those two faces of $2\left(m_{1}+m_{2}-2\right)$ sides each with $2\left(m_{1}+m_{2}-2\right)$ quadrilaterals, once the required edges are added over the tube. Now,

$$
\begin{aligned}
E^{(2)} & =\left(m_{1}-1\right) m_{2}+\left(m_{2}-1\right) m_{1} \\
& =2 m_{1} m_{2}-m_{1}-m_{2}
\end{aligned}
$$

so that

$$
\begin{aligned}
E^{(3)} & =m_{3} E^{(2)}+\left(m_{3}-1\right) V^{(2)} \\
& =3 m_{1} m_{2} m_{3}-m_{1} m_{3}-m_{2} m_{3}-m_{1} m_{2} .
\end{aligned}
$$

Also, $F^{(3)}=m_{3} F^{(2)}+\Delta F$, where $\Delta F$ is the increase in faces accounted for by the tubes we have added. This increase is of two types, corresponding to tubes attached within faces designated by (1) and to tubes attached within faces designated by (2). We have:

$$
\begin{aligned}
F^{(3)}= & m_{3}\left[\left(m_{1}-1\right)\left(m_{2}-1\right)+1\right]+2\left(\frac{m_{3}}{2}-1\right)\left(\frac{m_{1}}{2} \frac{m_{2}}{2}\right) \\
& +\frac{m_{3}}{2}\left[2\left(\frac{m_{1}}{2}-1\right)\left(\frac{m_{2}}{2}-1\right)+2\left(m_{1}+m_{2}-3\right)\right] \\
= & \frac{3}{2} m_{1} m_{2} m_{3}-\frac{m_{1} m_{2}}{2}-\frac{m_{1} m_{3}}{2}-\frac{m_{2} m_{3}}{2} .
\end{aligned}
$$

Furthermore, consider the set of faces obtained by taking, from each tube joining faces designated by (2), every second face. These faces are mutually vertex-disjoint, and contain all $m_{1} m_{2} m_{3}$ vertices of $H_{3}$. Now, form a second set of faces consisting of the remaining faces on the tubes joining faces designated by (2). These faces are also mutually vertex-disjoint, and contain all $m_{1} m_{2} m_{3}$ vertices of $H_{3}$. Moreover, the two sets of faces we have selected are clearly disjoint. Therefore, $S(3)$ is true.

Now we assume $S(n)$ to be true, and establish $S(n+1)$, for $n \geqq 3$. Given the graph $H_{n+1}$, we give the $m_{n+1}$ copies of $H_{n}$ minimal imbeddings as described by $S(n)$, 
with orientation as determined by the vertex set partition of $\boldsymbol{P}_{m_{n+1}}$. It is clear that we can make the required $\left(m_{n+1}-1\right)$ joins so as to obtain a quadrilateral imbedding for $H_{n+1}$. We have

$$
\begin{aligned}
E^{(n+1)} & =m_{n+1} E^{(n)}+\left(m_{n+1}-1\right) V^{(n)} \\
& =m_{n+1}\left(n M^{(n)}-M^{(n)} m^{(n)}\right)+\left(m_{n+1}-1\right) M^{(n)} \\
& =M^{(n+1)}\left(n+1-m^{(n+1)}\right) .
\end{aligned}
$$

Also, $F^{(n+1)}=m_{n+1} F^{(n)}+\Delta F$, where $\Delta F=\left(m_{n+1}-1\right)\left(\frac{1}{4} M^{(n)}\right)(2)$, where $m_{n+1}-1$ is the number of joins, $\frac{1}{4} M^{(n)}$ is the number of tubes per join, and there is a net increase in $F$ of two for each tube. We have

$$
\begin{aligned}
F^{(n+1)} & =m_{n+1}\left(M^{(n)} / 2\right)\left(n-m^{(n)}\right)+\frac{1}{2} M^{(n+1)}-\frac{1}{2} M^{(n)} \\
& =\left(M^{(n+1)} / 2\right)\left(n+1-m^{(n+1)}\right) .
\end{aligned}
$$

To complete the verification of $S(n+1)$, we must find two disjoint sets of $M^{(n+1)} / 4$ mutually vertex-disjoint quadrilateral faces each, both sets containing all $M^{(n+1)}$ vertices of $H_{n+1}$. We have two cases to consider:

Case (i). If $m_{n+1}$ is even we choose opposite faces on each tube of alternate joins to form one set, and the remaining faces on the same tubes to form the second set, as indicated in Figure 2.

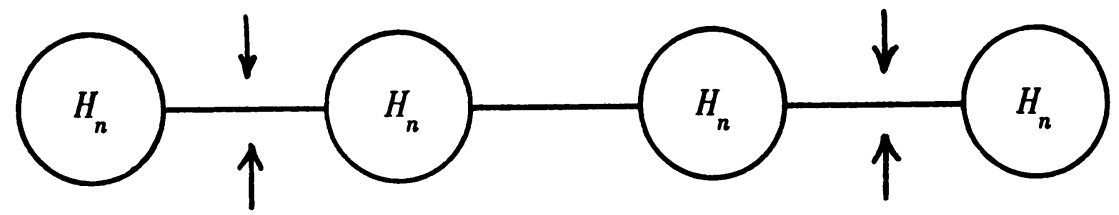

FIGURE 2. Selecting faces for $m_{n+1}$ even

Case (ii). If $m_{n+1}$ is odd, we make our selection as indicated in Figure 3, using at each end copy of $H_{n}$ the remaining set of $\frac{1}{4} M^{(n)}$ mutually vertex-disjoint quadrilaterals. As in Figure 2, an arrow at a join indicates that opposite faces on each tube of the join have been selected.

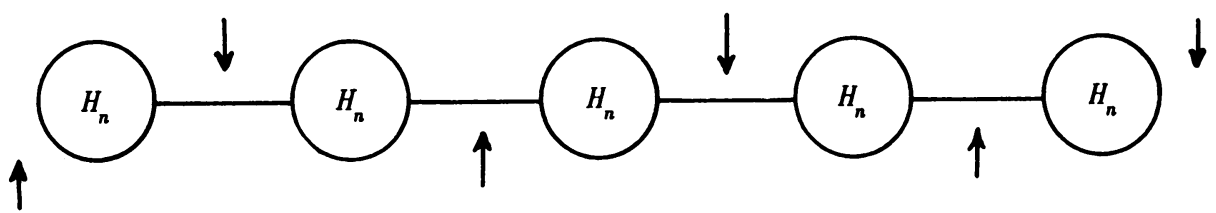

FIGURE 3. Selecting faces for $m_{n+1}$ odd

We have shown that $S(n+1)$ follows from $S(n)$, and hence that $S(n)$ holds for all $n \geqq 3$. It only remains to compute the genus of $H_{n}$. But by Proposition 2,

$$
\begin{aligned}
\gamma\left(H_{n}\right) & =1+\left(M^{(n)} / 4\right)\left(n-m^{(n)}\right)-M^{(n)} / 2 \\
& =1+\left(M^{(n)} / 4\right)\left(n-2-m^{(n)}\right) .
\end{aligned}
$$


Since the operation of taking the cartesian product is commutative, Theorem 4 can be applied if any three or more of the $m_{i}$ are even. Elementary probability considerations show that this fails to happen in only $\left(n^{2}+n+2\right) / 2^{n+1}$ of the possible cases, for fixed $n$. For $n>5$, this probability will be less than one half.

For the special case where $m_{i}=m, i=1, \ldots, n$, for $m$ even, we have:

COROLlaRY 4a. The genus of the graph $H_{n}^{(m)}$ is given by $\gamma\left(H_{n}^{(m)}\right)=1+\left(m^{n-1} / 4\right)$ $\cdot(m n-2 m-n)$, for $m$ any even positive integer.

Furthermore, if $m=2$ in the above formula, $H_{n}^{(2)}$ is the $n$-cube, since $P_{2}=K_{2}$, and we have the familiar result:

CoRollaRY 4b. $\gamma\left(Q_{n}\right)=1+2^{n-3}(n-4)$.

Repeated cartesian products of even cycles. Every even cycle is a bipartite graph, and the techniques of this paper apply also to the graph $G_{n}: G_{1}=C_{2 m_{1}}$, the cycle on $2 m_{1}$ vertices, and $G_{n}=G_{n-1} \times C_{2 m_{n}}$ for $n \geqq 2$. We require $m_{i} \geqq 2$, $i=1, \ldots, n$, for $C_{2}=K_{2}$ is not considered to be a cycle. Again let $M^{(n)}=\prod_{i=1}^{n} m_{i}$.

THEOREM 5. The genus of $G_{n}$ is given by $\gamma\left(G_{n}\right)=1+2^{n-2}(n-2) M^{(n)}$, for $n \geqq 2$.

Proof. By Proposition $1, G_{n}$ is a bipartite graph. We produce a quadrilateral imbedding for $G_{n}$, and compute $\gamma\left(G_{n}\right)$ using Proposition 2. For $G_{n}, V=2^{n} M^{(n)}$; and since $G_{n}$ is regular of degree $2 n$, it is a simple matter to compute $E=2^{n} n M^{(n)}$. Now, let the statement $S(n)$ be: There is an imbedding of $G_{n}$ for which $F^{(n)}=F_{4}^{(n)}$ $=n 2^{n-1} M^{(n)}$, including two disjoint sets of $2^{n-2} M^{(n)}$ mutually vertex-disjoint quadrilateral faces each, both sets containing all $2^{n} M^{(n)}$ vertices of $G_{n}$. We claim that $S(n)$ is true for all $n \geqq 2$. We verify this by induction.

That $S(2)$ is true is apparent from Figure 4, with the faces designated by (1) making up one set and those designated by (2) making up the other. Now we assume $S(n)$ to be true and establish $S(n+1)$, for $n \geqq 2$. For the graph $G_{n+1}$, we start with $2 m_{n+1}$ copies of $G_{n}$, minimally imbedded as described by $S(n)$. We partition the corresponding surfaces into $m_{n+1}$ copies of one orientation and

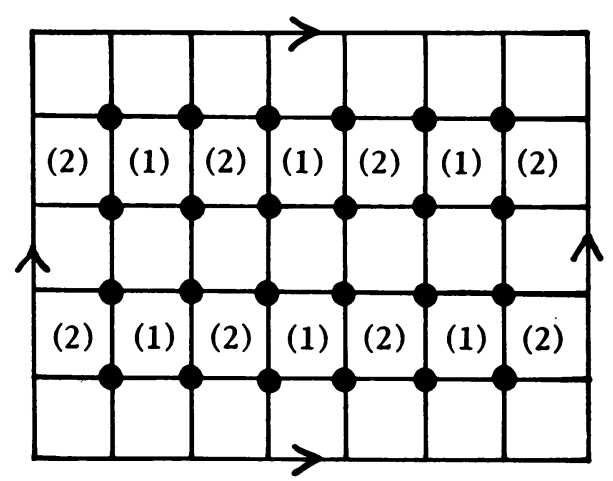

Figure 4. An imbedding of $C_{4} \times C_{6}$ in the torus 
$m_{n+1}$ copies of the reverse orientation, corresponding to the vertex set partition of $C_{2 m_{n+1}}$. From each copy, two joins must be made, both to copies of opposite orientation. From the statement $S(n)$, it is clear that these two joins can be made, each one over $2^{n-2} M^{(n)}$ tubes carrying four edges each. Each new face formed is a quadrilateral. In this fashion the required $2 m_{n+1}$ joins can be made to imbed $G_{n+1}$, with $F=F_{4}$. Now form one set of faces by selecting opposite quadrilaterals from each tube added in alternate joins in this construction. Form the second set by selecting the remaining quadrilaterals on the same tubes. It is clear that the two sets of faces thus selected are disjoint, and that each contains

$$
(2)\left(m_{n+1}\right)\left(2^{n-2} M^{(n)}\right)=2^{n-1} M^{(n+1)}
$$

mutually vertex-disjoint quadrilaterals; both sets contain all $2^{n+1} M^{(n+1)}$ vertices of $G_{n+1}$. Furthermore, $F^{(n+1)}=2 m_{n+1} F^{(n)}+\Delta F$, where $\Delta F=\left(2 m_{n+1}\right)\left(2^{n-2} M^{(n)}\right)(2)$, where $2 m_{n+1}$ joins have been made, with $2^{n-2} M^{(n)}$ tubes per join, and a net increase in $\boldsymbol{F}$ of 2 per tube. Hence,

$$
\begin{aligned}
F^{(n+1)} & =2 m_{n+1}\left(n 2^{n-1} M^{(n)}\right)+2^{n} M^{(n+1)} \\
& =(n+1) 2^{n} M^{(n+1)},
\end{aligned}
$$

and we have established that $S(n+1)$ follows from $S(n)$. Therefore, $S(n)$ holds, for all $n \geqq 2$. We can now compute:

$$
\begin{aligned}
\gamma\left(G_{n}\right) & =1+2^{n} n M^{(n)} / 4-2^{n} M^{(n)} / 2 \\
& =1+2^{n-2}(n-2) M^{(n)} .
\end{aligned}
$$

For the special case where $m_{i}=m, i=1, \ldots, n$, we have $M^{(n)}=m^{n}$, and:

COROllary 5a. The genus of $G_{n}^{(m)}$ is given by $\gamma\left(G_{n}^{(m)}\right)=1+2^{n-2}(n-2) m^{n}$.

Furthermore, if $m=2$ in the above formula, since $C_{4}=K_{2,2}=K_{2} \times K_{2}, G_{n}^{(2)}$ is the $2 n$-cube, and we obtain the familiar result:

COROLlaRY 5b. $\gamma\left(Q_{2 n}\right)=1+2^{2 n-2}(n-2)$.

Summary. The $n$-cube $Q_{n}$ is defined as a repeated cartesian product of the bipartite graph $K_{2}$ with itself. We have found the genus for generalizations of the $n$-cube in three different directions: in Theorem 3, regarding $K_{1,1}$ as $K_{2}$; in Theorem 4, regarding $P_{2}$ as $K_{2}$; and in Theorem 5, regarding $C_{4}$ as $K_{2} \times K_{2}$ to generalize the $2 n$-cube.

\section{REFERENCES}

1. L. W. Beineke and F. Harary, The genus of the n-cube, Canad. J. Math. 17 (1965), 494-496. MR 31 \#81.

2. J. Edmonds, A combinatorial representation for polyhedral surfaces, Notices Amer. Math. Soc. 7 (1960), 646. Abstract \#572-1.

3. F. Harary, Graph theory, Addison-Wesley, Reading, Mass., 1969. 
4. G. Ringel, Uber drei kombinatorische Probleme am n-dimensionalen Würfel und Würfelgitter, Abh. Math. Sem. Univ. Hamburg 20 (1955), 10-19. MR 17, 772.

5. —, Das Geschlecht des vollständigen paaren Graphen, Abh. Math. Sem. Univ. Hamburg 28 (1965), 139-150. MR 32 \#6439.

6. G. Ringel and J. W. T. Youngs, Das Geschlecht des Symmetrische Vollständige DreiFarbaren Graphen, Comment. Math. Helv. (to appear).

7. - Solution of the Heawood map-coloring problem, Proc. Nat. Acad. Sci. U.S.A. 60 (1968), 438-445. MR 37 \#3959.

8. A. T. White. The genus of the complete tripartite graph $K_{m n, n, n}$, J. Combinatorial Theory 7 (1969), 283-285.

9. J. W. T. Youngs, Minimal imbeddings and the genus of a graph, J. Math. Mech. 12 (1963), 303-315. MR 26 \#3043.

Michigan State University,

East Lansing, Michigan 48823

WeStern Michigan UNIVERSITY,

KalamazoO, Michigan 49001 